\title{
SHEARLET COORBIT SPACES: COMPACTLY SUPPORTED ANALYZING SHEARLETS, TRACES AND EMBEDDINGS
}

\author{
STEPHAN DAHLKE, GABRIELE STEIDL, AND GERD TESCHKE
}

\begin{abstract}
We show that compactly supported functions with sufficient smoothness and enough vanishing moments can serve as analyzing vectors for shearlet coorbit spaces. We use this approach to prove embedding theorems for subspaces of shearlet coorbit spaces resembling shearlets on the cone into Besov spaces. Furthermore, we show embedding relations of traces of these subspaces with respect to the real axes.
\end{abstract}

\section{INTRODUCTION}

One of the most important tasks in applied analysis is the analysis of signals which are usually modeled by (real or complex valued) functions. The first step is always to decompose the signal with respect to suitable building blocks. There are by now many different ways to choose these building blocks. Most prominent examples are the Fourier, the wavelet and the Gabor transform, respectively. All these different transforms have their advantages and drawback, which one to choose depends on the application and on the specific information one wants to extract from the signal. However, for many applications, in particular in image analysis, the wavelet transform is very often the method of choice. Indeed, wavelets are very well suited for piecewise smooth signals with isolated singularities, for in this case the wavelet expansion turns out to be quite sparse which gives rise to very efficient compression strategies. Unfortunately it has been observed that the detection of directional information by wavelets is difficult or at least not very efficient. Therefore, in recent years, much effort has been spent to design directional representation systems such as the curvelets [3], the ridgelets [2] and also the shearlets [14] (This list is clearly not complete). Among all these transforms, the shearlet transform stands out since it stems from a square-integrable group representation. This has been clarified in [5] where the underlying group, the full shearlet group, has been established. This pure group theoretical approach to shearlets has some important advantages. In particular, it is possible to derive canonical smoothness spaces associated with the shearlet transform. The basic tool to do this is provided by the coorbit space theory derived by Feichtinger and Gröchenig in a series of papers [8, 9, 10]. Under certain additional integrability conditions, the smoothness spaces related with a square-integrable group representation are defined by the decay of the associated voice transform. This technique is quite universal, and the classical smoothness spaces such as Besov and modulation spaces can be interpreted as coorbit spaces associated with the affine group and the Weyl-Heisenberg group, respectively. Moreover, the coorbit space theory provides a very general discretization technique which produces atomic decompositions and Banach frames for the coorbit spaces.

In [6], it has been clarified that the coorbit theory is indeed applicable to the full shearlet group. Moreover, in [7], a natural generalization to arbitrary space dimensions has been derived.

Date: today.

2000 Mathematics Subject Classification. 22D10, 42C15, 46E35, 47B25.

Key words and phrases. Compactly supported shearlets, coorbit space theory, square-integrable group representations, Banach frames, embedding of function spaces, Besov spaces, trace theorems. 
However, once these new smoothness spaces, the shearlet coorbit spaces, are established, some natural questions arise. How do these spaces really look like? Are there 'nice' sets of functions that are dense in these spaces? What are the relations to classical smoothness spaces such as Besov spaces? Do there exist embeddings into Besov spaces? And do there exist generalized versions of Sobolev embedding theorems for shearlet coorbit spaces? Moreover, can the associated trace spaces be identified? In this paper, we provide some first answers to these questions. We concentrate on the two-dimensional case, and we show that

- For large classes of weights, variants of Sobolev embeddings exist;

- for natural subclasses which in a certain sense correspond to the 'shearlets on the cone' [17], there exist embeddings into (homogeneous) Besov spaces:

- for the same subclass, the traces onto the coordinate axis can again be identified with homogeneous Besov spaces.

Our approach heavily relies on atomic decomposition techniques. Recall that the coorbit space theory naturally gives rise to Banach frames, and therefore, by using the associated norm equivalences, all the tasks outlined above can be studied by means of weighted sequence. In particular, based on the general analysis in [15], quite recently this technique has been applied to derive new embedding and trace results for Besov spaces [19]. The analysis presented in this paper was partially inspired by this thesis.

To make this approach really powerful, it is very convenient and sometimes even necessary to work with compactly supported building blocks. In the shearlet case, this is a nontrivial problem, since usually the analyzing shearlets are band-limited functions. For the specific case of shearlets on the cone, quite recently a first solution has been provided in [16]. We refer to the overview article [18] for a detailed discussion. Since the shearlets on the cone do not really fit into the group theoretical setting, we provide a new construction of families of compactly supported shearlets in this paper. We show that indeed a compactly supported function with sufficient smoothness and enough vanishing moments can serve as an analyzing vector for shearlet coorbit spaces.

This paper is organized as follows: We start by introducing the shearlet group in Section 2. In Section 3 we consider shearlet coorbit spaces, their atomic decompositions and shearlet Banach frames. We show that compactly supported shearlets can be used as analyzing vectors for these spaces, in particular there exist compactly supported shearlets within so-called 'better' sets used to define atomic decompositions. Finally we prove that the Schwartz functions are dense in our shearlet coorbit spaces. Section 4 deals with relations between shearlet coorbit spaces and Besov spaces. After recalling the general characteristics of homogeneous Besov spaces we prove embeddings of traces of certain subspaces of shearlet coorbit spaces on the real axes into (sums of) onedimensional Besov spaces. Finally, we show that these shearlet coorbit subspaces are themselves embedded into (sums of) Besov spaces of appropriate smoothness.

\section{The Shearlet Group}

In this section, we provide the basic notation and results about the shearlet group and its square integrable representations including the corresponding admissible functions, the so-called shearlets. For $a \in \mathbb{R}^{*}:=\mathbb{R} \backslash\{0\}$ and $s \in \mathbb{R}$, let

$$
A_{a}:=\left(\begin{array}{cc}
a & 0 \\
0 & \operatorname{sgn}(a) \sqrt{|a|}
\end{array}\right) \quad \text { and } \quad S_{s}:=\left(\begin{array}{ll}
1 & s \\
0 & 1
\end{array}\right)
$$

denote the parabolic scaling matrix and the shear matrix, respectively, where sgn $(a)$ denotes the sign of $a$. The (full) shearlet group $\mathbb{S}$ is defined to be the set $\mathbb{R}^{*} \times \mathbb{R} \times \mathbb{R}^{2}$ endowed with the group 
operation

$$
(a, s, t)\left(a^{\prime}, s^{\prime}, t^{\prime}\right)=\left(a a^{\prime}, s+s^{\prime} \sqrt{|a|}, t+S_{s} A_{a} t^{\prime}\right) .
$$

A left-invariant and right-invariant Haar measures of $\mathbb{S}$ is given by

$$
\mu_{\mathbb{S}, l}=\frac{d a}{|a|^{3}} d s d t \quad \text { and } \quad \mu_{\mathbb{S}, r}=\frac{d a}{|a|} d s d t,
$$

respectively and the modular function of $\mathbb{S}$ by $\triangle(a, s, t)=1 /|a|^{2}$. In the following, we use the left-invariant Haar measure $\mu_{\mathbb{S}}=\mu_{\mathbb{S}, l}$. Let $L_{x}, R_{x}$ denote the left and right translations by $x \in \mathbb{S}$, i.e., $L_{x} F(y):=F\left(x^{-1} y\right)$ and $R_{x} F(y):=F(y x)$.

Recall that a unitary representation of a locally compact group $G$ with the left-invariant Haar measure $\mu_{G}$ on a Hilbert space $\mathcal{H}$ is a homomorphism $\pi$ from $G$ into the group of unitary operators $\mathcal{U}(\mathcal{H})$ on $\mathcal{H}$ which is continuous with respect to the strong operator topology. For the shearlet group the mapping $\pi: \mathbb{S} \rightarrow \mathcal{U}\left(L_{2}\left(\mathbb{R}^{2}\right)\right)$ defined by

$$
\pi(a, s, t) \psi(x):=|a|^{-\frac{3}{4}} \psi\left(A_{a}^{-1} S_{s}^{-1}(x-t)\right)=|a|^{-\frac{3}{4}} \psi\left(\frac{1}{a}\left(x_{1}-t_{1}-s\left(x_{2}-t_{2}\right)\right), \frac{\operatorname{sgn} a}{\sqrt{|a|}}\left(x_{2}-t_{2}\right)\right)
$$

is a unitary representation of $\mathbb{S}$, see $[5,6]$. In the following, we use the abbreviation $\psi_{a, s, t}:=$ $\pi(a, s, t) \psi$. Let the Fourier transform be defined by

$$
\mathcal{F} f(\omega)=\hat{f}(\omega)=\int_{\mathbb{R}^{2}} f(x) e^{-2 \pi i\langle\omega, x\rangle} d x .
$$

Then straightforward computation yields

$$
\hat{\psi}_{a, s, t}(\omega)=|a|^{\frac{3}{4}} e^{-2 \pi i t \omega} \hat{\psi}\left(A_{a}^{\mathrm{T}} S_{s}^{\mathrm{T}} \omega\right)=|a|^{\frac{3}{4}} e^{-2 \pi i t \omega} \hat{\psi}\left(a \omega_{1}, \operatorname{sgn}(a) \sqrt{|a|}\left(s \omega_{1}+\omega_{2}\right)\right) .
$$

A function $\psi \in L_{2}\left(\mathbb{R}^{2}\right)$ is called admissible, if

$$
\int_{\mathbb{S}}|\langle\psi, \pi(g) \psi\rangle|^{2} d \mu_{\mathbb{S}}(g)<\infty
$$

If a unitary representation $\pi$ is irreducible and there exists at least one admissible function $\psi \in$ $L_{2}\left(\mathbb{R}^{2}\right)$ then $\pi$ is called square integrable.

The following result from [6] shows that the unitary representation $\pi$ defined in (1) is a squareintegrable representation of $\mathbb{S}$.

Theorem 2.1. A function $\psi \in L_{2}\left(\mathbb{R}^{2}\right)$ is admissible if and only if it fulfills the admissibility condition

$$
C_{\psi}:=\int_{\mathbb{R}} \int_{\mathbb{R}} \frac{\left|\hat{\psi}\left(\omega_{1}, \omega_{2}\right)\right|^{2}}{\omega_{1}^{2}} d \omega_{2} d \omega_{1}<\infty
$$

Then, for any $f \in L^{2}\left(\mathbb{R}^{2}\right)$, the following equality holds true:

$$
\int_{\mathbb{S}}\left|\left\langle f, \psi_{a, s, t}\right\rangle\right|^{2} d \mu_{\mathbb{S}}(a, s, t)=C_{\psi}\|f\|_{2}^{2} .
$$

In particular, the unitary representation $\pi$ is irreducible and hence square-integrable.

A function $\psi \in L_{2}\left(\mathbb{R}^{2}\right)$ fulfilling the admissibility condition (3) is called a continuous shearlet. The transform $\mathcal{S} \mathcal{H}_{\psi}: L_{2}\left(\mathbb{R}^{2}\right) \rightarrow L_{2}(\mathbb{S})$ defined by

$$
\mathcal{S} \mathcal{H}_{\psi} f(a, s, t)=\left\langle f, \psi_{a, s, t}\right\rangle
$$


and is called Continuous Shearlet Transform. The admissibility condition is important, since it implies a resolution of identity that allows the reconstruction of a function $f \in L_{2}\left(\mathbb{R}^{2}\right)$ from its Continuous Shearlet Transform.

\section{Shearlet Coorbit Spaces from Shearlets with Compact Support}

3.1. Shearlet Coorbit Spaces. Let $w$ be a positive, real-valued, continuous submultiplicative weight on $\mathbb{S}$, i.e., $w(g h) \leq w(g) w(h)$ for all $g, h \in \mathbb{S}$. To define our coorbit spaces we need the set

$$
\mathcal{A}_{w}:=\left\{\psi \in L_{2}\left(\mathbb{R}^{2}\right): \mathcal{S} \mathcal{H}_{\psi}(\psi)=\langle\psi, \pi(\cdot) \psi\rangle \in L_{1, w}\right\} .
$$

of analyzing vectors. In the following, we assume that our weight is symmetric with respect to the modular function, i.e., $w(g)=w\left(g^{-1}\right) \triangle\left(g^{-1}\right)$. Starting with an ordinary weight function $w$, its symmetric version can be obtained by $w^{\#}(g):=w(g)+w\left(g^{-1}\right) \triangle\left(g^{-1}\right)$. Moreover, it was proved in Lemma 2.4 of [8] that $\mathcal{A}_{w}=\mathcal{A}_{w^{\#}}$.

We want to show that $\mathcal{A}_{w}$ contains shearlets with compact support. To this end, we need the following auxiliary lemma which is a modification of Lemma 11.1.1 in [11].

Lemma 3.1. For $r>1$ and $\alpha>0$, the following estimate holds true

$$
I(x):=\int_{\mathbb{R}}(1+|t|)^{-r}(1+\alpha|x-t|)^{-r} d t \leq C\left(\frac{1}{\alpha}(1+|x|)^{-r}+(1+\alpha|x|)^{-r}\right) .
$$

Proof. Let

$$
\mathcal{N}_{x}:=\left\{t \in \mathbb{R}:|t-x| \leq \frac{|x|}{2}\right\}, \quad \mathcal{N}_{x}^{c}:=\left\{t \in \mathbb{R}:|t-x|>\frac{|x|}{2}\right\} .
$$

Then we obtain for $t \in \mathcal{N}_{x}$ by $|x|-|t| \leq|t-x| \leq|x| / 2$ that $|t| \geq|x| / 2$ and consequently

$$
(1+|t|)^{-r} \leq\left(1+\frac{|x|}{2}\right)^{-r} \leq 2^{r}(1+|x|)^{-r} .
$$

Now the above integral can be estimated as follows:

$$
\begin{aligned}
I(x) & =\int_{\mathcal{N}_{x}}(1+|t|)^{-r}(1+\alpha|x-t|)^{-r} d t+\int_{\mathcal{N}_{x}^{c}}(1+|t|)^{-r}(1+\alpha|x-t|)^{-r} d t \\
& \leq 2^{r}(1+|x|)^{-r} \int_{\mathcal{N}_{x}}(1+\alpha|x-t|)^{-r} d t+\left(1+\alpha \frac{|x|}{2}\right)^{-r} \int_{\mathcal{N}_{x}^{c}}(1+|t|)^{-r} d t \\
& \leq 2^{r} \frac{1}{\alpha}(1+|x|)^{-r} \int_{\mathbb{R}}(1+|u|)^{-r} d u+2^{r}(1+\alpha|x|)^{-r} \int_{\mathbb{R}}(1+|t|)^{-r} d t .
\end{aligned}
$$

This implies the assertion.

Let $Q_{D}:=[-D, D] \times[-D, D]$. The following theorem shows that $\mathcal{A}_{w}$ contains shearlets with compact support.

Theorem 3.2. Let $\psi(x) \in L_{2}\left(\mathbb{R}^{2}\right)$ fulfill $\operatorname{supp} \psi \in Q_{D}$. Suppose that the weight function satisfies $w(a, s, t)=w(a) \leq|a|^{-\rho_{1}}+|a|^{\rho_{2}}$ for $\rho_{1}, \rho_{2}>0$ and that

$$
\left|\hat{\psi}\left(\omega_{1}, \omega_{2}\right)\right| \leq C \frac{\left|\omega_{1}\right|^{n}}{\left(1+\left|\omega_{1}\right|\right)^{r}} \frac{1}{\left(1+\left|\omega_{2}\right|\right)^{r}}
$$

with $n \geq \max \left(\frac{1}{4}+\rho_{2}, \frac{9}{4}+\rho_{1}\right)$ and $r>n+\max \left(\frac{7}{4}+\rho_{2}, \frac{9}{4}+\rho_{1}\right)$. Then we have that $\mathcal{S H}_{\psi}(\psi) \in L_{1, w}(\mathbb{S})$, i.e.,

$$
I:=\int_{\mathbb{S}}\left|\mathcal{S H}_{\psi}(\psi)(g)\right| w(g) d \mu(g)<\infty .
$$


Proof. First we have by the support property of $\psi$ that $\mathcal{S H}_{\psi}(\psi)=\left\langle\psi, \psi_{a, s, t}\right\rangle \neq 0$ requires $\left(x_{1}, x_{2}\right) \in$ $Q_{D}$ and

$$
\begin{aligned}
-D & \leq \frac{\operatorname{sgn} a}{\sqrt{|a|}}\left(x_{2}-t_{2}\right) \leq D, \\
-D & \leq \frac{1}{a}\left(x_{1}-t_{1}-s\left(x_{2}-t_{2}\right)\right) \leq D .
\end{aligned}
$$

Hence $\left\langle\psi, \psi_{a, s, t}\right\rangle \neq 0$ implies that

$$
\begin{aligned}
-D(1+\sqrt{|a|}) & \leq t_{2} \leq D(1+\sqrt{|a|}) \\
-D(1+|a|+|s|(2+\sqrt{|a|})) & \leq t_{1} \leq D(1+|a|+|s|(2+\sqrt{|a|})) .
\end{aligned}
$$

Using this relation we obtain that

$$
I \leq \int_{\mathbb{R}^{*}} \int_{\mathbb{R}} 4 D^{2}(1+\sqrt{|a|})(1+|a|+|s|(2+\sqrt{|a|}))\left|\left\langle\psi, \psi_{a, s, t}\right\rangle\right| d s w(a) \frac{d a}{|a|^{3}} .
$$

Next, Plancherel's equality together with (2) and the decay assumptions on $\hat{\psi}$ yield

$$
\begin{aligned}
& I \leq C \int_{\mathbb{R}^{*}} \int_{\mathbb{R}}(1+\sqrt{|a|})(1+|a|+|s|(2+\sqrt{|a|}))\left|\left\langle\hat{\psi}, \hat{\psi}_{a, s, t}\right\rangle\right| d s w(a) \frac{d a}{|a|^{3}} \\
& \leq C \int_{\mathbb{R}^{*}} \int_{\mathbb{R}}(\underbrace{1+|a|^{\frac{1}{2}}+|a|+|a|^{\frac{3}{2}}}_{p_{3}\left(|a|^{\frac{1}{2}}\right)}+|s|(\underbrace{2+3|a|^{\frac{1}{2}}+a}_{p_{2}\left(|a|^{\frac{1}{2}}\right)})) J(a, s) d s w(a) \frac{d a}{|a|^{3}}
\end{aligned}
$$

where $\left|\mathcal{S H}_{\psi} \psi(a, s, t)\right| \leq J(a, s)$ and

$$
\begin{aligned}
J(a, s) & :=|a|^{\frac{3}{4}} \int_{\mathbb{R}} \int_{\mathbb{R}} \frac{\left|\omega_{1}\right|^{n}}{\left(1+\left|\omega_{1}\right|\right)^{r}} \frac{1}{\left(1+\left|\omega_{2}\right|\right)^{r}} \frac{\left|a \omega_{1}\right|^{n}}{\left(1+\left|a \omega_{1}\right|\right)^{r}} \frac{1}{\left(1+\sqrt{|a|}\left|s \omega_{1}+\omega_{2}\right|\right)^{r}} d \omega_{2} d \omega_{1} \\
& =\int_{\mathbb{R}} \frac{\left|\omega_{1}\right|^{n}}{\left(1+\left|\omega_{1}\right|\right)^{r}} \frac{\left|a \omega_{1}\right|^{n}}{\left(1+\left|a \omega_{1}\right|\right)^{r}} \int_{\mathbb{R}} \frac{1}{\left(1+\left|\omega_{2}\right|\right)^{r}} \frac{1}{\left(1+\sqrt{|a|}\left|s \omega_{1}+\omega_{2}\right|\right)^{r}} d \omega_{2} d \omega_{1} .
\end{aligned}
$$

The inner integral can be estimated by Lemma 3.1 which results in

$$
J(a, s) \leq C|a|^{n+\frac{3}{4}} \int_{\mathbb{R}} \frac{\left|\omega_{1}\right|^{n}}{\left(1+\left|\omega_{1}\right|\right)^{r}} \frac{\left|\omega_{1}\right|^{n}}{\left(1+\left|a \omega_{1}\right|\right)^{r}}\left(\frac{1}{\sqrt{|a|}\left(1+\left|s \omega_{1}\right|\right)^{r}}+\frac{1}{\left(1+\sqrt{|a|}\left|s \omega_{1}\right|\right)^{r}}\right) d \omega_{1} .
$$

Now we obtain

$$
\begin{aligned}
I \leq & C\left(\int_{\mathbb{R}^{*}} \int_{\mathbb{R}} \int_{\mathbb{R}}|a|^{n-\frac{11}{4}}\left(p_{3}+|s| p_{2}\right) \frac{\left|\omega_{1}\right|^{2 n}}{\left(1+\left|\omega_{1}\right|\right)^{r}\left(1+\left|a \omega_{1}\right|\right)^{r}} \frac{1}{\left(1+\left|s \omega_{1}\right|\right)^{r}} d s d \omega_{1} w(a) d a\right. \\
& \left.+\int_{\mathbb{R}^{*}} \int_{\mathbb{R}} \int_{\mathbb{R}}|a|^{n-\frac{9}{4}}\left(p_{3}+|s| p_{2}\right) \frac{\left|\omega_{1}\right|^{2 n}}{\left(1+\left|\omega_{1}\right|\right)^{r}\left(1+\left|a \omega_{1}\right|\right)^{r}} \frac{1}{\left(1+\sqrt{|a|}\left|s \omega_{1}\right|\right)^{r}} d s d \omega_{1} w(a) d a\right) .
\end{aligned}
$$


Since the integrand is even in $\omega_{1}, s$ and $a$ this can be further simplified as

$$
\begin{aligned}
I \leq & C\left(\int_{0}^{\infty} a^{n-\frac{11}{4}} p_{3}(\sqrt{a}) \int_{0}^{\infty} \frac{\omega_{1}^{2 n}}{\left(1+\omega_{1}\right)^{r}\left(1+a \omega_{1}\right)^{r}} \int_{0}^{\infty} \frac{1}{\left(1+s \omega_{1}\right)^{r}} d s d \omega_{1} w(a) d a\right. \\
& +\int_{0}^{\infty} a^{n-\frac{11}{4}} p_{2}(\sqrt{a}) \int_{0}^{\infty} \frac{\omega_{1}^{2 n}}{\left(1+\omega_{1}\right)^{r}\left(1+a \omega_{1}\right)^{r}} \int_{0}^{\infty} \frac{s}{\left(1+s \omega_{1}\right)^{r}} d s d \omega_{1} w(a) d a \\
& +\int_{0}^{\infty} a^{n-\frac{9}{4}} p_{3}(\sqrt{a}) \int_{0}^{\infty} \frac{\omega_{1}^{2 n}}{\left(1+\omega_{1}\right)^{r}\left(1+a \omega_{1}\right)^{r}} \int_{0}^{\infty} \frac{1}{\left(1+\sqrt{a} s \omega_{1}\right)^{r}} d s d \omega_{1} w(a) d a \\
& \left.+\int_{0}^{\infty} a^{n-\frac{9}{4}} p_{2}(\sqrt{a}) \int_{0}^{\infty} \frac{\omega_{1}^{2 n}}{\left(1+\omega_{1}\right)^{r}\left(1+a \omega_{1}\right)^{r}} \int_{0}^{\infty} \frac{s}{\left(1+\sqrt{a} s \omega_{1}\right)^{r}} d s d \omega_{1} w(a) d a\right) .
\end{aligned}
$$

Substituting $t:=s \omega_{1}$ with $d t=\omega_{1} d s$ in the first two integrals and $t:=\sqrt{a} s \omega_{1}$ with $d t=\sqrt{a} \omega_{1} d s$ in the last two integrals, we obtain for $r>2$ that

$$
\begin{aligned}
I \leq & C\left(\int_{0}^{\infty} \frac{\omega_{1}^{2 n-1}}{\left(1+\omega_{1}\right)^{r}} \int_{0}^{\infty} a^{n-\frac{11}{4}} p_{3}(\sqrt{a}) \frac{1}{\left(1+a \omega_{1}\right)^{r}} w(a) d a d \omega_{1}\right. \\
& +\int_{0}^{\infty} \frac{\omega_{1}^{2 n-2}}{\left(1+\omega_{1}\right)^{r}} \int_{0}^{\infty} a^{n-\frac{11}{4}} p_{2}(\sqrt{a}) \frac{1}{\left(1+a \omega_{1}\right)^{r}} w(a) d a d \omega_{1} \\
& +\int_{0}^{\infty} \frac{\omega_{1}^{2 n-1}}{\left(1+\omega_{1}\right)^{r}} \int_{0}^{\infty} a^{n-\frac{11}{4}} p_{3}(\sqrt{a}) \frac{1}{\left(1+a \omega_{1}\right)^{r}} w(a) d a d \omega_{1} \\
& \left.+\int_{0}^{\infty} \frac{\omega_{1}^{2 n-2}}{\left(1+\omega_{1}\right)^{r}} \int_{0}^{\infty} a^{n-\frac{13}{4}} p_{2}(\sqrt{a}) \frac{1}{\left(1+a \omega_{1}\right)^{r}} w(a) d a d \omega_{1}\right) .
\end{aligned}
$$

Substituting $b:=a \omega_{1}$ with $d b=\omega_{1} d a$ and bounding $w$ accordingly we conclude further that

$$
\begin{aligned}
I \leq & C\left(\int_{0}^{\infty} \frac{\omega_{1}^{n+\frac{3}{4}+\rho_{1}}}{\left(1+\omega_{1}\right)^{r}} \int_{0}^{\infty} p_{3}\left(\sqrt{\frac{b}{\omega_{1}}}\right) \frac{b^{n-\frac{11}{4}-\rho_{1}}}{(1+b)^{r}} d b d \omega_{1}\right. \\
& +\int_{0}^{\infty} \frac{\omega_{1}^{n-\frac{1}{4}+\rho_{1}}}{\left(1+\omega_{1}\right)^{r}} \int_{0}^{\infty} p_{2}\left(\sqrt{\frac{b}{\omega_{1}}}\right) \frac{b^{n-\frac{11}{4}-\rho_{1}}}{(1+b)^{r}} d b d \omega_{1} \\
& +\int_{0}^{\infty} \frac{\omega_{1}^{n+\frac{1}{4}+\rho_{1}}}{\left(1+\omega_{1}\right)^{r}} \int_{0}^{\infty} p_{2}\left(\sqrt{\frac{b}{\omega_{1}}}\right) \frac{b^{n-\frac{13}{4}-\rho_{1}}}{(1+b)^{r}} d b d \omega_{1} \\
& +\int_{0}^{\infty} \frac{\omega_{1}^{n+\frac{3}{4}-\rho_{2}}}{\left(1+\omega_{1}\right)^{r}} \int_{0}^{\infty} p_{3}\left(\sqrt{\frac{b}{\omega_{1}}}\right) \frac{b^{n-\frac{11}{4}+\rho_{2}}}{(1+b)^{r}} d b d \omega_{1} \\
& +\int_{0}^{\infty} \frac{\omega_{1}^{n-\frac{1}{4}-\rho_{2}}}{\left(1+\omega_{1}\right)^{r}} \int_{0}^{\infty} p_{2}\left(\sqrt{\frac{b}{\omega_{1}}}\right) \frac{b^{n-\frac{11}{4}+\rho_{2}}}{(1+b)^{r}} d b d \omega_{1} \\
& \left.+\int_{0}^{\infty} \frac{\omega_{1}^{n+\frac{1}{4}-\rho_{2}}}{\left(1+\omega_{1}\right)^{r}} \int_{0}^{\infty} p_{2}\left(\sqrt{\frac{b}{\omega_{1}}}\right) \frac{b^{n-\frac{13}{4}+\rho_{2}}}{(1+b)^{r}} d b d \omega_{1}\right) .
\end{aligned}
$$

Regarding that $p_{k} \in \Pi_{k}, k=2,3$ we see that the integrals are finite if $n \geq \max \left(\frac{1}{4}+\rho_{2}, \frac{9}{4}+\rho_{1}\right)$ and $r>n+\max \left(\frac{7}{4}+\rho_{2}, \frac{9}{4}+\rho_{1}\right)$. This finishes the proof. 
For an analyzing vector $\psi$ we can consider the space

$$
\mathcal{H}_{1, w}:=\left\{f \in L_{2}\left(\mathbb{R}^{2}\right): \mathcal{S H}_{\psi}(f)=\langle f, \pi(\cdot) \psi\rangle \in L_{1, w}(\mathbb{S})\right\}
$$

with norm $\|f\|_{\mathcal{H}_{1, w}}:=\left\|\mathcal{S} \mathcal{H}_{\psi} f\right\|_{L_{1, w}(\mathbb{S})}$ and its anti-dual $\mathcal{H}_{1, w}^{\sim}$, the space of all continuous conjugatelinear functionals on $\mathcal{H}_{1, w}$. The spaces $\mathcal{H}_{1, w}$ and $\mathcal{H}_{1, w}^{\sim}$ are $\pi$-invariant Banach spaces with continuous embedding $\mathcal{H}_{1, w} \hookrightarrow \mathcal{H} \hookrightarrow \mathcal{H}_{1, w}^{\sim}$. Then the inner product on $L_{2}\left(\mathbb{R}^{2}\right) \times L_{2}\left(\mathbb{R}^{2}\right)$ extends to a sesquilinear form on $\mathcal{H}_{1, w}^{\sim} \times \mathcal{H}_{1, w}$, therefore for $\psi \in \mathcal{H}_{1, w}$ and $f \in \mathcal{H}_{1, w}^{\sim}$ the extended representation coefficients

$$
\mathcal{S H}_{\psi}(f)(a, s, t):=\langle f, \pi(a, s, t) \psi\rangle_{\mathcal{H}_{1, w} \times \mathcal{H}_{1, w}}
$$

are well-defined.

Let $m$ be a $w$-moderate weight on $\mathbb{S}$ which means that $m(x y z) \leq w(x) m(y) w(z)$ for all $x, y, z \in \mathbb{S}$. For $1 \leq p \leq \infty$, let

$$
L_{p, m}(\mathbb{S}):=\left\{F \text { measurable }: F m \in L_{p}(\mathbb{S})\right\} .
$$

We are interested in the following Banach spaces which are called shearlet coorbit spaces

$$
\mathcal{S C}_{p, m}:=\left\{f \in \mathcal{H}_{1, w}^{\sim}: \mathcal{S H}_{\psi}(f) \in L_{p, m}(\mathbb{S})\right\}, \quad\|f\|_{\mathcal{S C}_{p, m}}:=\left\|\mathcal{S H}_{\psi} f\right\|_{L_{p, m}(\mathbb{S})} .
$$

Note that the definition of $\mathcal{S C}_{p, m}$ is independent of the analyzing vector $\psi$ and of the weight $w$ in the sense that $\tilde{w}$ with $w(g) \leq C \tilde{w}(g)$ for all $g \in \mathbb{S}$ and $\mathcal{A}_{\tilde{w}} \neq\{0\}$ give rise to the same space see [8, Theorem 4.2].

In applications, one may start with some sub-multiplicative weight $m$ and use the symmetric weight $w:=m^{\#}$ for the definition of $\mathcal{A}_{w}$. Obviously, we have that $m$ is $w$-moderate.

3.2. Atomic Decompositions and Shearlet Banach Frames. To construct atomic decompositions and Banach frames of our shearlet coorbit spaces the following better subset $\mathcal{B}_{w}$ of $\mathcal{A}_{w}$ has to be non-empty

$$
\mathcal{B}_{w}:=\left\{\psi \in L_{2}\left(\mathbb{R}^{2}\right): \mathcal{S H}_{\psi}(\psi) \in \mathcal{W}\left(C_{0}, L_{1, w}\right)\right\},
$$

where $\mathcal{W}\left(C_{0}, L_{1, w}\right)$ is the Wiener-Amalgam space

$$
\mathcal{W}\left(C_{0}, L_{1, w}\right):=\left\{F:\left\|\left(L_{x} \chi_{\mathcal{Q}}\right) F\right\|_{\infty} \in L_{1, w}\right\}, \quad\left\|\left(L_{x} \chi_{\mathcal{Q}}\right) F\right\|_{\infty}=\sup _{y \in x \mathcal{Q}}|F(y)|
$$

and $\mathcal{Q}$ is a relatively compact neighborhood of the identity element in $\mathbb{S}$, see [13]. Note that in general $\mathcal{B}_{w}$ is defined with respect to the right version $\left.\mathcal{W}^{R}\left(C_{0}, L_{1, w}\right)\right):=\left\{F:\left\|\left(R_{x} \chi_{Q}\right) F\right\|_{\infty}=\right.$ $\left.\sup _{y \in \mathcal{Q} x^{-1}}|F(y)| \in L_{1, w}\right\}$ of the Wiener-Amalgam space. Regarding that $\mathcal{S H}_{\psi}(\psi)(g)=\mathcal{S H}_{\psi} \psi\left(g^{-1}\right)$ and assuming that $\mathcal{Q}=\mathcal{Q}^{-1}$ both definitions of $\mathcal{B}_{w}$ coincide.

Corollary 3.3. Let $\psi(x) \in L_{2}\left(\mathbb{R}^{2}\right)$ fulfill $\operatorname{supp} \psi \in Q_{D}$. Suppose that the weight function satisfies $w(a, s, t)=w(a) \leq|a|^{-\rho_{1}}+|a|^{\rho_{2}}$ for $\rho_{1}, \rho_{2}>0$ and that

$$
\left|\hat{\psi}\left(\omega_{1}, \omega_{2}\right)\right| \leq C \frac{\left|\omega_{1}\right|^{n}}{\left(1+\left|\omega_{1}\right|\right)^{r}} \frac{1}{\left(1+\left|\omega_{2}\right|\right)^{r}}
$$

for sufficiently large $n$ and $r$. Then we have that $\psi \in \mathcal{B}_{w}$.

Proof. To keep technicalities at a reasonable level, we restrict ourselves to the case $w \equiv 1$. Let $\mathcal{Q}=\mathcal{Q}^{-1} \subset\left[\frac{1}{\alpha}, \alpha\right] \times[-\sigma, \sigma] \times Q_{\tau}$, where $\alpha>1, \sigma, \tau>0$. In the following, we restrict our attention to group elements of $\mathbb{S}$ with $a>0$ and $s \geq 0$. The other cases can be deduced in a similar way. Let $\left(a_{q}, s_{q}, t_{q}\right) \in \mathcal{Q}$ and

$$
\left(a^{\prime}, s^{\prime}, t^{\prime}\right):=(a, s, t)\left(a_{q}, s_{q}, t_{q}\right)=\left(a a_{q}, s+s_{q} \sqrt{a},\left(\begin{array}{c}
t_{1}+a t_{q, 1}+\sqrt{a} s t_{q, 2} \\
t_{2}+\sqrt{a} t_{q, 2}
\end{array}\right)\right) .
$$


We are interested in

$$
G(a, s, t):=\sup _{\left(a_{q}, s_{q}, t_{q}\right) \in \mathcal{Q}}\left|\mathcal{S} \mathcal{H}_{\psi} \psi\left(a^{\prime}, s^{\prime}, t^{\prime}\right)\right| .
$$

As in the proof of Theorem 3.2 we have that $\mathcal{S H}_{\psi} \psi\left(a^{\prime}, s^{\prime}, t^{\prime}\right)$ is zero if $t^{\prime}$ does not fulfill

$$
\begin{aligned}
& -D \leq \frac{1}{\sqrt{a^{\prime}}}\left(x_{2}-t_{2}^{\prime}\right) \leq D, \\
& -D \leq \frac{1}{a^{\prime}}\left(x_{1}-t_{1}^{\prime}-s^{\prime}\left(x_{2}-t_{2}^{\prime}\right)\right) \leq D,
\end{aligned}
$$

where $x \in Q_{D}$. By definition of $a^{\prime}, s^{\prime}, t^{\prime}$ this implies that

$$
\begin{aligned}
x_{2}-\sqrt{a} t_{q, 2}-D \sqrt{a_{q} a} & \leq t_{2} \leq x_{2}-\sqrt{a} t_{q, 2}+D \sqrt{a_{q} a}, \\
r-D a a_{q} & \leq t_{1} \leq r+D a a_{q},
\end{aligned}
$$

where $r:=x_{1}-a t_{q, 1}-\sqrt{a} s t_{q, 2}-\left(s+s_{q} \sqrt{a}\right)\left(x_{2}-t_{2}-\sqrt{a} t_{q, 2}\right.$. By definition of $\mathcal{Q}$ we conclude that $G(a, s, t)$ becomes zero if $t$ is not contained in

$$
\begin{aligned}
-C(1+\sqrt{a}) & \leq t_{2} \leq C(1+\sqrt{a}), \quad C:=\max \{D, D(\sqrt{\alpha}+\tau\}, \\
-P_{2}(\sqrt{a})-s P_{1}(\sqrt{a}) & \leq t_{1} \leq P_{2}(\sqrt{a})+s P_{1}(\sqrt{a}),
\end{aligned}
$$

where $P_{k} \in \Pi_{k}$ are polynomials with nonnegative coefficients depending on $\alpha, \sigma$ and $\tau$. As in the proof of Theorem 3.2 we conclude that $\left|\mathcal{S H}_{\psi} \psi\left(a^{\prime}, s^{\prime}, t^{\prime}\right)\right| \leq C J\left(a^{\prime}, s^{\prime}\right)$, where

$$
\begin{aligned}
J\left(a^{\prime}, s^{\prime}\right):= & \left(a^{\prime}\right)^{n+\frac{3}{4}} \int_{\mathbb{R}} \frac{\left|\omega_{1}\right|^{2 n}}{\left(1+\left|\omega_{1}\right|\right)^{r}\left(1+\left|a^{\prime} \omega_{1}\right|\right)^{r}}\left(\frac{1}{\sqrt{a^{\prime}}\left(1+\left|s^{\prime} \omega_{1}\right|\right)^{r}}+\frac{1}{\left(1+\sqrt{a^{\prime}}\left|s^{\prime} \omega_{1}\right|\right)^{r}}\right) d \omega_{1} \\
= & \left(a a_{q}\right)^{n+\frac{3}{4}} \int_{\mathbb{R}} \frac{\left|\omega_{1}\right|^{2 n}}{\left(1+\left|\omega_{1}\right|\right)^{r}\left(1+\left|a a_{q} \omega_{1}\right|\right)^{r}}\left(\frac{1}{\sqrt{a a_{q}}\left(1+\left|s+s_{q} \sqrt{a}\right|\left|\omega_{1}\right|\right)^{r}}\right) d \omega_{1} \\
& +\left(a a_{q}\right)^{n+\frac{3}{4}} \int_{\mathbb{R}} \frac{\left|\omega_{1}\right|^{2 n}}{\left(1+\left|\omega_{1}\right|\right)^{r}\left(1+\left|a a_{q} \omega_{1}\right|\right)^{r}}\left(\frac{1}{\left(1+\sqrt{a a_{q}}\left|s+s_{q} \sqrt{a}\right|\left|\omega_{1}\right|\right)^{r}}\right) d \omega_{1} .
\end{aligned}
$$

For $0 \leq s \leq 2 \sigma \sqrt{a}$ we use the estimate $\left|s+s_{q} \sqrt{a}\right| \geq 0$ to get

$$
|G(a, s, t)| \leq C a^{n+\frac{3}{4}}\left(\frac{1}{\sqrt{a}}+1\right) \int_{\mathbb{R}} \frac{\left|\omega_{1}\right|^{2 n}}{\left(1+\left|\omega_{1}\right|\right)^{r}\left(\frac{1}{\alpha}+\left|a \omega_{1}\right|\right)^{r}} d \omega_{1} .
$$

For $s>2 \sigma \sqrt{a}$ we have that $\left|s+s_{q} \sqrt{a}\right| \geq|s| / 2$ and consequently

$$
|G(a, s, t)| \leq C a^{n+\frac{3}{4}} \int_{\mathbb{R}} \frac{\left|\omega_{1}\right|^{2 n}}{\left(1+\left|\omega_{1}\right|\right)^{r}\left(\frac{1}{\alpha}+\left|a \omega_{1}\right|\right)^{r}}\left(\frac{1}{\sqrt{a}\left(2+|s|\left|\omega_{1}\right|\right)^{r}}+\frac{1}{\left(\frac{2}{\alpha}+\sqrt{a}|s|\left|\omega_{1}\right|\right)^{r}}\right) d \omega_{1} .
$$

If the following integral is finite, then we can conclude that $G \in L_{1, w}$ :

$$
\begin{aligned}
I:= & \int_{\mathbb{S}}|G(a, s, t)| d t d s \frac{d a}{|a|^{3}} \\
\leq & C \int_{0}^{\infty} \int_{0}^{2 \sigma \sqrt{a}}\left(p_{3}(\sqrt{a})+s p_{2}(\sqrt{a})\right)|G(a, s, t)| d s \frac{d a}{a^{3}} \\
& +C \int_{0}^{\infty} \int_{2 \sigma \sqrt{a}}^{\infty}\left(p_{3}(\sqrt{a})+s p_{2}(\sqrt{a})\right)|G(a, s, t)| d s \frac{d a}{a^{3}},
\end{aligned}
$$


where $p_{3}(\sqrt{a}):=(1+\sqrt{a}) P_{2}(\sqrt{a}) \in \Pi_{3}$ and $p_{2}(\sqrt{a}):=(1+\sqrt{a}) P_{1}(\sqrt{a}) \in \Pi_{2}$. By the above estimates of $G$ this can be rewritten as

$$
\begin{aligned}
I \leq & C \int_{0}^{\infty} \int_{0}^{\infty} a^{n-\frac{9}{4}}\left(p_{3}(\sqrt{a})+\tilde{p}_{3}(\sqrt{a})\right)(\sqrt{a}+1) \frac{\omega_{1}^{2 n}}{\left(1+\omega_{1}\right)^{r}\left(\frac{1}{\alpha}+a \omega_{1}\right)^{r}} d a d \omega_{1} \\
& +C \int_{0}^{\infty} \int_{0}^{\infty} \int_{0}^{\infty} a^{n-\frac{11}{4}}\left(p_{3}(\sqrt{a})+s p_{2}(\sqrt{a})\right) \frac{\omega_{1}^{2 n}}{\left(1+\omega_{1}\right)^{r}\left(\frac{1}{\alpha}+a \omega_{1}\right)^{r}} \frac{1}{\left(2+s \omega_{1}\right)^{r}} d a d s d \omega_{1} \\
& +C \int_{0}^{\infty} \int_{0}^{\infty} \int_{0}^{\infty} a^{n-\frac{9}{4}}\left(p_{3}(\sqrt{a})+s p_{2}(\sqrt{a})\right) \frac{1}{\left(1+\omega_{1}\right)^{r}\left(\frac{1}{\alpha}+a \omega_{1}\right)^{r}} \frac{1}{\left(\frac{2}{\alpha}+\sqrt{a} s \omega_{1}\right)^{r}} d a d s d \omega_{1},
\end{aligned}
$$

where $\tilde{p}_{3}(\sqrt{a}):=2 \sigma \sqrt{a} p_{2}(\sqrt{a}) \in \Pi_{3}$. The two later integrals can be estimated as in the proof of Theorem 3.2 with $\rho_{1}=\rho_{2}=0$ and the first integral by

$$
I_{1} \leq C \int_{0}^{\infty} \frac{\omega_{1}^{n+\frac{5}{4}}}{\left(1+\omega_{1}\right)^{r}} \int_{0}^{\infty} p_{4}\left(\sqrt{\frac{b}{\omega_{1}}}\right) \frac{b^{n-\frac{9}{4}}}{\left(\frac{1}{\alpha}+b\right)^{r}} d b d \omega_{1},
$$

where $p_{4}(\sqrt{a})=\left(p_{3}(\sqrt{a})+\tilde{p}_{3}(\sqrt{a})\right)(\sqrt{a}+1)$. This integral is finite for $n \geq \frac{9}{4}$ and $r>n+\frac{9}{4}$. This completes the proof.

A (countable) family $X=\left\{\left(a_{i}, s_{i}, t_{i}\right): i \in \mathcal{I}\right\}$ in $\mathbb{S}$ is said to be $U$-dense if $\cup_{i \in \mathcal{I}}\left(a_{i}, s_{i}, t_{i}\right) U=\mathbb{S}$, and separated if for some compact neighborhood $Q$ of $e$ we have $\left(a_{i}, s_{i}, t_{i}\right) Q \cap\left(a_{j}, s_{j}, t_{j}\right) Q=\emptyset, i \neq j$, and relatively separated if $X$ is a finite union of separated sets. Let $\alpha>1$ and $\sigma, \tau>0$ be defined such that

$$
\left[\frac{1}{\alpha}, \alpha\right) \times[-\sigma, \sigma) \times Q_{\tau} \subset U
$$

Then it was shown in [6] that the set

$$
X:=\left\{\left(\epsilon \alpha^{-j}, \sigma \alpha^{-j / 2} k, S_{\sigma \alpha^{-j / 2} k} A_{\alpha^{-j}} \tau l\right): j \in \mathbb{Z}, k \in \mathbb{Z}, l \in \mathbb{Z}^{2}, \epsilon \in\{-1,1\}\right\}
$$

is $U$-dense and relatively separated. For $g \in \mathbb{S}$, let

$$
\operatorname{osc}_{U}(g):=\sup _{u \in U}\left|\mathcal{S H}_{\Psi} \psi(u g)-\mathcal{S H}_{\Psi} \psi(g)\right| .
$$

The following theorem from [6] shows that the functions in our shearlet coorbit spaces possess atomic decompositions.

Theorem 3.4. Assume that the irreducible, unitary representation $\pi$ is $w$-integrable and let an appropriately normalized $\psi \in \mathcal{B}_{w}$. Choose a neighborhood $U$ of e so small that

$$
\left\|\operatorname{osc}_{U}\right\|_{L_{1, w}(\mathbb{S})}<1 .
$$

Then for any $U$-dense and relatively separated set $X=\left\{\left(a_{i}, s_{i}, t_{i}\right): i \in \mathcal{I}\right\}$ the space $\mathcal{S C}_{p, m}$ has the following atomic decomposition: If $f \in \mathcal{S C}_{p, m}$, then

$$
f=\sum_{i \in \mathcal{I}} c_{i}(f) \pi\left(a_{i}, s_{i}, t_{i}\right) \psi
$$

where the sequence of coefficients depends linearly on $f$ and satisfies

$$
\left\|\left(c_{i}(f)\right)_{i \in \mathcal{I}}\right\|_{\ell_{p, m}} \leq C\|f\|_{\mathcal{S C} \mathcal{C}_{p, m}}
$$

with a constant $C$ depending only on $\psi$ and with $\ell_{p, m}$ being defined by

$$
\ell_{p, m}:=\left\{c=\left(c_{i}\right)_{i \in \mathcal{I}}:\|c\|_{\ell_{p, m}}:=\|c m\|_{\ell_{p}}<\infty\right\}
$$


where $m=\left(m\left(a_{i}, s_{i}, t_{i}\right)\right)_{i \in \mathcal{I}}$. Conversely, if $\left(c_{i}(f)\right)_{i \in \mathcal{I}} \in \ell_{p, m}$, then $f=\sum_{i \in \mathcal{I}} c_{i} \pi\left(a_{i}, s_{i}, t_{i}\right) \psi$ is in $\mathcal{S C}_{p, m}$ and

$$
\|f\|_{\mathcal{S C}_{p, m}} \leq C^{\prime}\left\|\left(c_{i}(f)\right)_{i \in \mathcal{I}}\right\|_{\ell_{p, m}}
$$

Given such an atomic decomposition, the problem arises under which conditions a function $f$ is completely determined by its moments $\left\langle f, \pi\left(a_{i}, s_{i}, t_{i}\right) \psi\right\rangle, i \in \mathcal{I}$ and how $f$ can be reconstructed from these moments. This is answered by the following theorem from [6] which establishes the existence of Banach frames.

Theorem 3.5. Impose the same assumptions as in Theorem 3.4. Choose a neighborhood $U$ of $e$ such that

$$
\left\|\operatorname{osc}_{U}\right\|_{L_{1, w}(\mathbb{S})}<1 /\left\|\mathcal{S H}_{\psi}(\psi)\right\|_{L_{1, w}(\mathbb{S})} .
$$

Then, for every $U$-dense and relatively separated family $X=\left\{\left(a_{i}, s_{i}, t_{i}\right): i \in \mathcal{I}\right\}$ in $\mathbb{S}$ the set $\left\{\pi\left(a_{i}, s_{i}, t_{i}\right) \psi: i \in \mathcal{I}\right\}$ is a Banach frame for $\mathcal{S C}_{p, m}$. This means that

i) $f \in \mathcal{S C}_{p, m}$ if and only if $\left(\left\langle f, \pi\left(a_{i}, s_{i}, t_{i}\right) \psi\right\rangle_{\mathcal{H}_{1, w}^{\sim} \times \mathcal{H}_{1, w}}\right)_{i \in \mathcal{I}} \in \ell_{p, m}$,

ii) there exist two constants $0<D \leq D^{\prime}<\infty$ such that

$$
D\|f\|_{\mathcal{S C}_{p, m}} \leq\left\|\left(\left\langle f, \pi\left(a_{i}, s_{i}, t_{i}\right) \psi\right\rangle_{\mathcal{H}_{1, w}^{\sim} \times \mathcal{H}_{1, w}}\right)_{i \in \mathcal{I}}\right\|_{\ell_{p, w}} \leq D^{\prime}\|f\|_{\mathcal{S C}_{p, m}},
$$

iii) there exists a bounded, linear reconstruction operator $\mathcal{R}$ from $\ell_{p, m}$ to $\mathcal{S C}_{p, m}$ such that $\mathcal{R}\left(\left(\left\langle f, \psi\left(a_{i}, s_{i}, t_{i}\right) \psi\right\rangle_{\mathcal{H}_{1, w}^{\sim} \times \mathcal{H}_{1, w}}\right)_{i \in \mathcal{I}}\right)=f$.

\section{Structure of Shearlet Coorbit Spaces}

4.1. Atomic decomposition of Besov spaces. Let us recall the characterization of homogeneous Besov spaces $B_{p, q}^{\sigma}$ from [12], see also [15, 20]. For inhomogeneous Besov spaces we refer to [19]. For $\alpha>1, D>1$ and $K \in \mathbb{N}_{0}$, a $K$ times differentiable function $a$ on $\mathbb{R}^{d}$ is called a $K$-atom if the following two conditions are fulfilled:

A1) $\operatorname{supp} a \subset D Q_{j, m}\left(\mathbb{R}^{d}\right)$ for some $m \in \mathbb{R}^{d}$, where $Q_{j, m}\left(\mathbb{R}^{d}\right)$ denotes the cube in $\mathbb{R}^{d}$ centered at $\alpha^{-j} m$ with sides parallel to the coordinate axes and side length $2 \alpha^{-j}$.

A2) $\left|D^{\gamma} a(x)\right| \leq \alpha^{|\gamma| j}$ for $|\gamma| \leq K$.

Now the homogeneous Besov spaces can be characterized as follows.

Theorem 4.1. Let $D>1$ and $K \in \mathbb{N}_{0}$ with $K \geq 1+\lfloor\sigma\rfloor$ be fixed. Let $1 \leq p \leq \infty$. Then $f \in B_{p, q}^{\sigma}$ if and only if it can be represented as

$$
f(x)=\sum_{j \in Z} \sum_{l \in \mathbb{Z}^{d}} \lambda(j, l) a_{j, l}(x),
$$

where the $a_{j, l}$ are $K$-atoms with $\operatorname{supp} a_{j, l} \subset D Q_{j, l}\left(\mathbb{R}^{d}\right)$ and

$$
\|f\|_{B_{p, q}^{\sigma}} \sim \inf \left(\sum_{j \in \mathbb{Z}} \alpha^{j\left(\sigma-\frac{d}{p}\right) q}\left(\sum_{l \in \mathbb{Z}^{d}}|\lambda(j, l)|^{p}\right)^{\frac{q}{p}}\right)^{\frac{1}{q}}
$$

where the infimum is taken over all admissible representations (19). 
In this section, we are mainly interested in weights

$$
m(a, s, t)=m(a):=|a|^{-r}, r \geq 0
$$

and use the abbreviation

$$
\mathcal{S C}_{p, r}:=\mathcal{S C}_{p, m}
$$

For simplicity, we further assume in the following that we can use $\sigma=\tau=1$ in the $U$-dense, relatively separated set (12) and restrict ourselves to the case $\epsilon=1$. In other words, we assume that $f \in \mathcal{S C}_{p, r}$ can be written as

$$
\begin{aligned}
f(x) & =\sum_{j \in \mathbb{Z}} \sum_{k \in \mathbb{Z}} \sum_{l \in \mathbb{Z}^{2}} c(j, k, l) \pi\left(\alpha^{-j}, \sigma \alpha^{-j / 2} k, S_{\alpha^{-j / 2} k} A_{\alpha^{-j}} l\right) \psi(x) \\
& =\sum_{j \in \mathbb{Z}} \sum_{k \in \mathbb{Z}} \sum_{l \in \mathbb{Z}^{2}} c(j, k, l) \alpha^{\frac{3}{4} j} \psi\left(\alpha^{j} x_{1}-\alpha^{j / 2} k x_{2}-l_{1}, \alpha^{j / 2} x_{2}-l_{2}\right) .
\end{aligned}
$$

To derive reasonable trace and embedding theorems, it is necessary to introduce the following subspaces of $\mathcal{S C}_{p, r}$. For fixed $\psi \in B_{w}$ we denote by $\mathcal{S C C}_{p, r}$ be the closed subspace of $\mathcal{S C}_{p, r}$ consisting of those functions which are representable as in (20) but with integers $|k| \leq \alpha^{j / 2}$. As we shall see in the sequel for each of these $\psi$ the resulting spaces $\mathcal{S C C}_{p, r}$ embed in the same scale of Besov spaces, and the same holds true for the trace theorems.

4.2. A Density Result. In most of the classical smoothness spaces like Sobolev and Besov spaces dense subsets of 'nice' functions can be identified. Typically, the set of Schwartz functions $\mathcal{S}$ serves as such a dense subset. We refer to [1] and any book of Hans Triebel for further information. By the following theorem the same is true for our shearlet coorbit spaces.

Theorem 4.2. Let

$$
\mathcal{S}_{0}:=\left\{f \in \mathcal{S}:|\hat{f}(\omega)| \leq \frac{\omega_{1}^{2 \alpha}}{\left(1+\|\omega\|^{2}\right)^{2 \alpha}} \forall \alpha>0\right\}
$$

and $m(a, s, t)=m(a, s):=|a|^{r}\left(\frac{1}{|a|}+|a|+|s|\right)^{n}$ for some $r \in \mathbb{R}, n \geq 0$. Then the set of Schwartz functions forms a dense subset of the shearlet coorbit space $\mathcal{S} C_{p, m}$.

Proof. As in [6, Theorem 4.7] it can be shown that $\mathcal{S}_{0}$ is at least contained in $\mathcal{S} C_{p, m}$. (Note that in [6] the weight $\left(\frac{1}{|a|}+|a|\right)^{r}\left(\frac{1}{|a|}+|a|+|s|\right)^{n}, r, n>0$ which is not smaller than 1 was considered.) It remains to show the density. To this end, we observe from Theorem 4.2 in [6] that certain bandlimited Schwartz functions can be used as analyzing shearlets. Now let us recall that the atomic decomposition in (14) has to be understood as a limit of finite linear combinations with respect to the shearlet coorbit norm. However, every finite linear combination of Schwartz functions is again a Schwartz function, hence (14) implies that we have found for any $f \in \mathcal{S} C_{p, m}$ a sequence of Schwartz functions which converges to $f$.

4.3. Traces on the Real Axes. In this subsection, we investigate the traces of functions lying in certain subspaces of $\mathcal{S C}_{p, r}$ with respect to the horizontal and vertical axes, respectively.

Theorem 4.3. Let $T r_{h} f$ denote the restriction of $f$ to the (horizontal) $x_{1}$-axis, i.e., $\left(\operatorname{Tr}_{h} f\right)\left(x_{1}\right):=$ $f\left(x_{1}, 0\right)$. Then $\operatorname{Tr}_{h}\left(\mathcal{S C C}_{p, r}\right) \subset B_{p, p}^{\sigma_{1}}(\mathbb{R})+B_{p, p}^{\sigma_{2}}(\mathbb{R})$, where

$$
B_{p, p}^{\sigma_{1}}(\mathbb{R})+B_{p, p}^{\sigma_{2}}(\mathbb{R}):=\left\{h \mid h=h_{1}+h_{2}, h_{1} \in B_{p, p}^{\sigma_{1}}(\mathbb{R}), h_{2} \in B_{p, p}^{\sigma_{2}}(\mathbb{R})\right\}
$$

and the parameters $\sigma_{1}$ and $\sigma_{2}$ satisfy the conditions

$$
\sigma_{1}=r-\frac{5}{4}+\frac{3}{2 p}, \quad \sigma_{2}=r-\frac{3}{4}+\frac{1}{p} .
$$


Note that $\sigma_{1} \leq \sigma_{2}$ for $p \geq 2$.

Proof. Using (20) we split $f$ into $f=f_{1}+f_{2}$, where

$$
\begin{aligned}
f_{1}\left(x_{1}, x_{2}\right) & :=\sum_{j \geq 0} \sum_{|k| \leq \alpha^{j / 2}} \sum_{l \in \mathbb{Z}^{2}} c(j, k, l) \alpha^{\frac{3}{4} j} \psi\left(\alpha^{j} x_{1}-\alpha^{j / 2} k x_{2}-l_{1}, \alpha^{j / 2} x_{2}-l_{2}\right), \\
f_{2}\left(x_{1}, x_{2}\right) & :=\sum_{j<0} \sum_{l \in \mathbb{Z}^{2}} c(j, 0, l) \alpha^{\frac{3}{4} j} \psi\left(\alpha^{j} x_{1}-l_{1}, \alpha^{j / 2} x_{2}-l_{2}\right),
\end{aligned}
$$

By Theorem 3.3 we can choose $\psi$ compactly supported in $[-D, D] \times[-D, D]$ for some $D>1$. Moreover, we can assume that $\left|D_{1}^{\gamma} \psi\right| \leq 1$ for $0 \leq \gamma \leq K:=\max \left\{K_{1}, K_{2}\right\}$, where $K_{1}:=1+\left\lfloor\sigma_{1}\right\rfloor$, $K_{2}:=1+\left\lfloor\sigma_{2}\right\rfloor$ and where $D_{1} \psi$ denotes the derivative with respect to the first component of $\psi$. Now $\operatorname{Tr}_{h} f$ can be written as

$$
\begin{aligned}
\operatorname{Tr}_{h} f\left(x_{1}\right)=f\left(x_{1}, 0\right) & =\sum_{j \in \mathbb{Z}} \sum_{|k| \leq \alpha^{j / 2}} \sum_{l \in \mathbb{Z}^{2}} c(j, k, l) \alpha^{\frac{3}{4} j} \psi\left(\alpha^{j} x_{1}-l_{1},-l_{2}\right) \\
& =\sum_{j \in \mathbb{Z}} \sum_{l_{1} \in \mathbb{Z}} \sum_{|k| \leq \alpha^{j / 2}} \sum_{\left|l_{2}\right| \leq D} c\left(j, k, l_{1}, l_{2}\right) \alpha^{\frac{3}{4} j} \psi\left(\alpha^{j} x_{1}-l_{1},-l_{2}\right) \\
& =\sum_{j \geq 0} \sum_{l_{1} \in \mathbb{Z}} \lambda\left(j, l_{1}\right) a_{j, l_{1}}\left(x_{1}\right)+\sum_{j<0} \sum_{l_{1} \in \mathbb{Z}} \lambda\left(j, l_{1}\right) a_{j, l_{1}}\left(x_{1}\right) \\
& =\operatorname{Tr}_{h} f_{1}\left(x_{1}\right)+T r_{h} f_{2}\left(x_{1}\right),
\end{aligned}
$$

where for $j \geq 0$,

$$
\begin{aligned}
a_{j, l_{1}}\left(x_{1}\right) & := \begin{cases}\lambda\left(j, l_{1}\right)^{-1} \alpha^{\frac{3}{4} j} \sum_{|k| \leq \alpha^{j / 2}} \sum_{\left|l_{2}\right| \leq D} c\left(j, k, l_{1}, l_{2}\right) \psi\left(\alpha^{j} x_{1}-l_{1},-l_{2}\right) & \text { if } \lambda\left(j, l_{1}\right) \neq 0, \\
0 & \text { otherwise },\end{cases} \\
\lambda\left(j, l_{1}\right) & :=\alpha^{\frac{3}{4} j} \sum_{|k| \leq \alpha^{j / 2}} \sum_{\left|l_{2}\right| \leq D}\left|c\left(j, k, l_{1}, l_{2}\right)\right|,
\end{aligned}
$$

and for $j<0$

$$
\begin{aligned}
a_{j, l_{1}}\left(x_{1}\right) & := \begin{cases}\lambda\left(j, l_{1}\right)^{-1} \alpha^{\frac{3}{4} j} \sum_{\left|l_{2}\right| \leq D} c\left(j, 0, l_{1}, l_{2}\right) \psi\left(\alpha^{j} x_{1}-l_{1},-l_{2}\right) & \text { if } \lambda\left(j, l_{1}\right) \neq 0, \\
0 & \text { otherwise }\end{cases} \\
\lambda\left(j, l_{1}\right) & :=\alpha^{\frac{3}{4} j} \sum_{\left|l_{2}\right| \leq D}\left|c\left(j, 0, l_{1}, l_{2}\right)\right| .
\end{aligned}
$$

We have that $\operatorname{supp} \psi\left(\alpha^{j} x_{1}-l_{1},-l_{2}\right) \subset D Q_{j, l_{1}}(\mathbb{R})$ which is also true for all $a_{j, l_{1}}$ and by construction we know further that $\left|D^{\gamma} a_{j, l_{1}}\right| \leq \alpha^{j \gamma}, 0 \leq \gamma \leq K$. Thus, the $a_{j, l_{1}}$ are $K_{1}$-atoms on $\mathbb{R}$. Next, we consider

$$
\begin{aligned}
\left\|\operatorname{Tr}_{h} f_{1}\right\|_{B_{p, p}^{\sigma_{1}}} & \lesssim\left(\sum_{j \in \mathbb{Z}} \alpha^{j\left(\sigma_{1}-\frac{1}{p}\right) p} \sum_{l_{1} \in \mathbb{Z}}\left|\lambda\left(j, l_{1}\right)\right|^{p}\right)^{\frac{1}{p}} \\
& =\left(\sum_{j \geq 0} \alpha^{j p\left(\sigma_{1}+\frac{3}{4}-\frac{1}{p}\right)} \sum_{l_{1} \in \mathbb{Z}}\left(\sum_{|k| \leq \alpha^{j / 2}} \sum_{\left|l_{2}\right| \leq D}\left|c\left(j, k, l_{1}, l_{2}\right)\right|\right)^{p}\right)^{\frac{1}{p}} .
\end{aligned}
$$


Since $\left(\sum_{i=1}^{N}\left|z_{i}\right|\right)^{p} \leq N^{p-1} \sum_{i=1}^{N}\left|z_{i}\right|^{p}$ and the set $\left\{k \in \mathbb{Z}:|k| \leq \alpha^{j / 2}\right\}$ contains $C \alpha^{j / 2}$ elements we can estimate

$$
\begin{aligned}
\left\|T r_{h} f_{1}\right\|_{B_{p, p}^{\sigma}} & \lesssim\left(\sum_{j \geq 0} \alpha^{j p\left(\sigma_{1}+\frac{5}{4}-\frac{3}{2 p}\right)} \sum_{|k| \leq \alpha^{j / 2}} \sum_{l \in \mathbb{R}^{2}}|c(j, k, l)|^{p}\right)^{\frac{1}{p}} \\
& \lesssim\left(\sum_{j \in \mathbb{Z}} \alpha^{j p r} \sum_{k \in \mathbb{Z}} \sum_{l \in \mathbb{R}^{2}}|c(j, k, l)|^{p}\right)^{\frac{1}{p}} \lesssim\|f\|_{\mathcal{S} \mathcal{C}_{p, r}}
\end{aligned}
$$

with $r=\sigma_{1}+\frac{5}{4}-\frac{3}{2 p}$. In the same way we obtain that

$$
\begin{aligned}
\left\|T r_{h} f_{2}\right\|_{B_{p, p}^{\sigma_{2}}} & \lesssim\left(\sum_{j<0} \alpha^{j p\left(\sigma_{2}+\frac{3}{4}-\frac{1}{p}\right)} \sum_{l \in \mathbb{R}^{2}}|c(j, 0, l)|^{p}\right)^{\frac{1}{p}} \\
& \lesssim\left(\sum_{j \in \mathbb{Z}} \alpha^{j p r} \sum_{k \in \mathbb{Z}} \sum_{l \in \mathbb{R}^{2}}|c(j, k, l)|^{p}\right)^{\frac{1}{p}} \lesssim\|f\|_{\mathcal{S} \mathcal{C}_{p, r}}
\end{aligned}
$$

with $r=\sigma_{2}+\frac{3}{4}-\frac{1}{p}$. This completes the proof.

By the following corollary the restriction to $\mathcal{S C C}_{p, r}$ is not necessary for $p=1$.

Corollary 4.4. For $p=1$, the embedding $\operatorname{Tr}_{h}\left(\mathcal{S C}_{1, r}\right) \subset B_{1,1}^{\sigma}(\mathbb{R})$ with $\sigma=r-\frac{3}{4}+\frac{1}{p}$ holds true.

Proof. Following the lines of the previous proof, where the summation with respect to $k$ is over $\mathbb{Z}$, we obtain

$$
\left\|T r_{h} f\right\|_{B_{1,1}^{\sigma}} \lesssim \sum_{j \in \mathbb{Z}} \alpha^{j\left(\left(\sigma+\frac{3}{4}\right) p-1\right)} \sum_{l_{1} \in \mathbb{Z}} \sum_{k \in \mathbb{Z}} \sum_{\left|l_{2}\right| \leq D}\left|c\left(j, k, l_{1}, l_{2}\right)\right| \leq C\|f\|_{\mathcal{S C}_{1, r}}
$$

with $r=\sigma+\frac{3}{4}-\frac{1}{p}$ and we are done.

Let us turn to traces on the vertical axis.

Theorem 4.5. Let $T r_{v} f$ denote the restriction of $f$ to the (vertical) $x_{2}$-axis, i.e., $\left(\operatorname{Tr}_{v} f\right)\left(x_{2}\right):=$ $f\left(0, x_{2}\right)$. Then the embedding $\operatorname{Tr}_{v}\left(\mathcal{S C C}_{p, r}\right) \subset B_{p, p}^{\sigma_{1}}(\mathbb{R})+B_{p, p}^{\sigma_{2}}(\mathbb{R})$, holds true, where $\sigma_{1}$ is the largest number such that

$$
\sigma_{1}+\left\lfloor\sigma_{1}\right\rfloor \leq 2 r-\frac{9}{2}+\frac{3}{p}, \quad \text { and } \quad \sigma_{2}=2 r-\frac{3}{2}+\frac{1}{p} .
$$

Proof. As in (21) and(21) we split $f$ into $f=f_{1}+f_{2}$, where we can choose $\psi$ compactly supported in $[-D, D] \times[-D, D]$ for some $D>1$ and normalized such that the derivatives of order $0 \leq \gamma \leq K$ with $K:=\max \left\{K_{1}, K_{2}\right\}$, where $K_{1}:=1+\left\lfloor\sigma_{1}\right\rfloor, K_{2}:=1+\left\lfloor\sigma_{2}\right\rfloor$ are not larger than 1 . By the support assumption on $\psi$ we have that

$$
\begin{aligned}
\alpha^{-j / 2}\left(l_{2}-D\right) & \leq x_{2} \leq \alpha^{-j / 2}\left(l_{2}+D\right), \\
-k l_{2}-D(1+|k|) & \leq l_{1} \leq-k l_{2}+D(1+|k|) .
\end{aligned}
$$

Let $I_{k, l_{2}}:=\left\{r \in \mathbb{Z}:\left|r+k m_{2}\right| \leq D(1+|k|)\right\}$. Now we obtain that

$$
\operatorname{Tr}_{v} f\left(x_{2}\right)=f\left(0, x_{2}\right)=\sum_{j \in \mathbb{Z}} \sum_{|k| \leq \alpha^{j / 2}} \sum_{l \in \mathbb{Z}^{2}} c(j, k, l) \alpha^{\frac{3}{4} j} \psi\left(-\alpha^{j / 2} k x_{2}-l_{1}, \alpha^{j / 2} x_{2}-l_{2}\right) .
$$

This can be rewritten as

$$
\begin{aligned}
f\left(0, x_{2}\right) & =\sum_{j \geq 0} \sum_{l_{2} \in \mathbb{Z}} \lambda\left(j, l_{2}\right) a_{j, l_{2}}\left(x_{2}\right)+\sum_{j<0} \sum_{l_{2} \in \mathbb{Z}} \lambda\left(j, l_{2}\right) a_{j, l_{2}}\left(x_{2}\right) \\
& =\operatorname{Tr}_{v} f_{1}\left(x_{2}\right)+T r_{v} f_{2}\left(x_{2}\right),
\end{aligned}
$$


where for $j \geq 0$,

$$
a_{j, l_{2}}\left(x_{2}\right):=\lambda\left(j, l_{2}\right)^{-1} \alpha^{\frac{3+2 K_{1}}{4} j} \sum_{|k| \leq \alpha^{j / 2}} \sum_{l_{1} \in I_{k, l_{2}}} c\left(j, k, l_{1}, l_{2}\right) \alpha^{-K_{1} j / 2} \psi\left(-\alpha^{j / 2} k x_{2}-l_{1}, \alpha^{j / 2} x_{2}-l_{2}\right)
$$

if $\lambda\left(j, l_{2}\right) \neq 0$ and $a_{j, l_{2}}\left(x_{2}\right)=0$ otherwise and

$$
\lambda\left(j, l_{2}\right):=\alpha^{\frac{3+2 K_{1}}{4} j} \sum_{|k| \leq \alpha^{j / 2}} \sum_{l_{1} \in I_{k, l_{2}}}\left|c\left(j, k, l_{1}, l_{2}\right)\right|
$$

and for $j<0$,

$$
a_{j, l_{2}}\left(x_{2}\right):=\lambda\left(j, l_{2}\right)^{-1} \alpha^{\frac{3}{4} j} \sum_{\left|l_{1}\right| \leq D} c\left(j, 0, l_{1}, l_{2}\right) \psi\left(-l_{1}, \alpha^{j / 2} x_{2}-l_{2}\right)
$$

if $\lambda\left(j, l_{2}\right) \neq 0$ and $a_{j, l_{2}}\left(x_{2}\right)=0$ otherwise and

$$
\lambda\left(j, l_{2}\right):=\alpha^{\frac{3}{4} j} \sum_{\left|l_{1}\right| \leq D}\left|c\left(j, 0, l_{1}, l_{2}\right)\right| .
$$

We have that $\operatorname{supp} \psi\left(-\alpha^{j / 2} k x_{2}-l_{1}, \alpha^{j / 2} x_{2}-l_{2}\right) \subset D Q_{j, l_{2}}(\mathbb{R})$, where the cube is considered with respect to $\sqrt{\alpha}$ now. This is also true for $a_{j, l_{2}}$. For $j \geq 0$ we conclude by $|k| \leq \alpha^{j / 2}$ that $\alpha^{-K j / 2}\left|D^{\gamma} \psi\left(-\alpha^{j / 2} k x_{2}-l_{1}, \alpha^{j / 2} x_{2}-l_{2}\right)\right| \leq \alpha^{\frac{j}{2} \gamma}$ and consequently $\left|D^{\gamma} a_{j, l_{2}}\right| \leq \alpha^{\frac{j}{2} \gamma}, \gamma \leq K_{1}$. For $j<0$ we also have that $\left|D^{\gamma} a_{j, l_{2}}\right| \leq \alpha^{\frac{j}{2} \gamma}$. Thus $a_{j, l_{2}}$ are $K_{1}$-atoms. We get

$$
\begin{aligned}
\left\|T r_{v} f_{1}\right\|_{B_{p, p} \sigma_{1}} & \lesssim\left(\sum_{j \in \mathbb{Z}} \alpha^{\frac{j}{2}\left(\sigma_{1}-\frac{1}{p}\right) p} \sum_{l_{2} \in \mathbb{Z}}\left|\lambda\left(j, l_{2}\right)\right|^{p}\right)^{\frac{1}{p}} \\
& \leq\left(\sum_{j \geq 0} \alpha^{\frac{j}{2}\left(\sigma_{1}-\frac{1}{p}\right) p} \alpha^{\frac{j}{2}\left(\frac{3+2 K_{1}}{2}\right) p} \alpha^{\frac{j}{2}\left(2-\frac{2}{p}\right) p} \sum_{|k| \leq \alpha^{j / 2} / \in \mathbb{R}^{2}}|c(j, k, l)|^{p}\right)^{\frac{1}{p}} \\
& \leq\left(\sum_{j \in \mathbb{Z}} \alpha^{\frac{j}{2}\left(\sigma_{1}+\frac{7}{2}+K_{1}-\frac{3}{p}\right) p} \sum_{|k| \leq \alpha^{j / 2}} \sum_{l \in \mathbb{R}^{2}}|c(j, k, l)|^{p}\right)^{\frac{1}{p}} \\
& \leq\left(\sum_{j \in \mathbb{Z}} \alpha^{\frac{j}{2}\left(\sigma_{1}+\frac{7}{2}+1+\left\lfloor\sigma_{1}\right\rfloor-\frac{3}{p}\right) p} \sum_{|k| \leq \alpha^{j / 2}} \sum_{l \in \mathbb{R}^{2}}|c(j, k, l)|^{p}\right)^{\frac{1}{p}} \\
& \leq\left(\sum_{j \in \mathbb{Z}} \alpha^{j p r} \sum_{k \in \mathbb{Z}} \sum_{l \in \mathbb{R}^{2}}|c(j, k, l)|^{p}\right)^{\frac{1}{p}} \\
& \lesssim\|f\|_{\mathcal{S} \mathcal{C}_{p, r}}
\end{aligned}
$$


with $r \geq \frac{1}{2}\left(\sigma_{1}+\left\lfloor\sigma_{1}\right\rfloor+\frac{9}{2}-\frac{3}{p}\right)$. Analogously we can compute

$$
\begin{aligned}
\left\|T r_{v} f_{2}\right\|_{B_{p, p} \sigma_{2}} & \lesssim\left(\sum_{j \in \mathbb{Z}} \alpha^{\frac{j}{2}\left(\sigma_{2}-\frac{1}{p}\right) p} \sum_{l_{2} \in \mathbb{Z}}\left|\lambda\left(j, l_{2}\right)\right|^{p}\right)^{\frac{1}{p}} \\
& \leq\left(\sum_{j<0} \alpha^{\frac{j}{2}\left(\sigma_{2}-\frac{1}{p}+\frac{3}{2}\right) p} \sum_{l \in \mathbb{R}^{2}}|c(j, 0, l)|^{p}\right)^{\frac{1}{p}} \\
& \leq\left(\sum_{j \in \mathbb{Z}} \alpha^{j p r} \sum_{k \in \mathbb{Z}} \sum_{l \in \mathbb{R}^{2}}|c(j, k, l)|^{p}\right)^{\frac{1}{p}} \\
& \lesssim\|f\|_{\mathcal{S C}, r}
\end{aligned}
$$

with $r=\frac{1}{2}\left(\sigma_{2}+\frac{3}{2}-\frac{1}{p}\right)$ and we are done.

4.4. Embedding Results. In this subsection, we prove embedding results of certain subspaces of shearlet coorbit spaces into (sums of) homogeneous Besov spaces. But first we provide a result concerning the embedding within shearlet coorbit spaces. In [8, Section 5.7] some embedding theorems for general $L_{p, m}$ coorbit spaces were given. In particular, the authors mentioned that for a fixed weight $m$, these spaces are monotonically increasing with $p$. The following corollary is a special results in this direction.

Corollary 4.6. For $1 \leq p_{1} \leq p_{2} \leq \infty$ the embedding $\mathcal{S C}_{p_{1}, r} \subset \mathcal{S C}_{p_{2}, r}$ holds true. Introducing the 'smoothness spaces' $\mathcal{G}_{p}^{r}:=\mathcal{S C}_{p, r+d\left(\frac{1}{2}-\frac{1}{p}\right)}$. this implies the continuous embedding

$$
\mathcal{G}_{p_{1}}^{r_{1}} \subset \mathcal{G}_{p_{2}}^{r_{2}}, \quad \text { if } \quad r_{1}-\frac{d}{p_{1}}=r_{2}-\frac{d}{p_{2}} .
$$

For convenience we add the simple proof.

Proof. By Theorem 3.4 we obtain that

$$
\|f\|_{\mathcal{S C}_{p_{2}, r}} \lesssim \|\left(c_{\epsilon}(j, k, l) \|_{\ell_{p_{2}, r}} \lesssim\left(\sum_{j \in \mathbb{Z}} \alpha^{j r p_{2}} \sum_{\substack{k, l \\ \epsilon \in\{-1,1\}}}\left|c_{\epsilon}(j, k, l)\right|^{p_{2}}\right)^{\frac{1}{p_{2}}},\right.
$$

where $c_{\epsilon}(j, k, l)$ is the coefficient belonging in the representation (14) with respect to (12) to the function $\pi\left(\epsilon \alpha^{-j}, \sigma \alpha^{-j / 2} k, S_{\sigma \alpha^{-j / 2} k} A_{\alpha^{-j}} \tau l\right) \psi$. Since $\ell_{p_{1}} \subset \ell_{p_{2}}$ for $p_{1} \leq p_{2}$ we get finally that

$$
\begin{aligned}
\|f\|_{\mathcal{S C}_{p_{2}, r}} & \lesssim\left(\sum_{j \in \mathbb{Z}} \alpha^{j r p_{2}}\left(\sum_{\substack{k, l \\
\epsilon \in\{-1,1\}}}\left|c_{\epsilon}(j, k, l)\right|^{p_{1}}\right)^{\frac{p_{2}}{p_{1}}}\right)^{\frac{1}{p_{2}}} \\
& \lesssim\left(\sum_{j \in \mathbb{Z}} \alpha^{j r p_{1}} \sum_{\substack{k, l \\
\epsilon \in\{-1,1\}}}\left|c_{\epsilon}(j, k, l)\right|^{p_{1}}\right)^{\frac{1}{p_{1}}} \lesssim\|f\|_{\mathcal{S C}_{p_{1}, r} .}
\end{aligned}
$$

Next we state our final result.

Theorem 4.7. The embedding $\mathcal{S C C}_{p, r} \subset B_{p, p}^{\sigma_{1}}\left(\mathbb{R}^{2}\right)+B_{p, p}^{\sigma_{2}}\left(\mathbb{R}^{2}\right)$, holds true, where $\sigma_{1}$ is the largest number such that

$$
\sigma_{1}+\left\lfloor\sigma_{1}\right\rfloor \leq 2 r-\frac{9}{2}+\frac{4}{p}, \quad \text { and } \quad \sigma_{2}-\frac{\left\lfloor\sigma_{2}\right\rfloor}{2}=r+\frac{3}{2 p}+\frac{1}{4}
$$


Proof. By (20) we know that $f \in \mathcal{S C C}_{p, r}$ can be written as

$$
f(x)=\sum_{j \in \mathbb{Z}} \sum_{|k| \leq \alpha^{j / 2}} \sum_{l \in \mathbb{Z}^{2}} c(j, k, l) \alpha^{\frac{3}{4} j} \psi\left(\alpha^{j} x_{1}-\alpha^{j / 2} k x_{2}-l_{1}, \alpha^{j / 2} x_{2}-l_{2}\right),
$$

where we can choose $\psi$ compactly supported in $[-D, D] \times[-D, D]$ for some $D>1$ and normalized such that the derivatives of order $0 \leq|\gamma| \leq K:=\max \left\{K_{1}, K_{2}\right\}, K_{1}:=1+\left\lfloor\sigma_{1}\right\rfloor, K_{2}:=1+\left\lfloor\sigma_{2}\right\rfloor$ are not larger than 1.

We split $f \in \mathcal{S C C}_{p, r}$ as in (21) and (22) into $f_{1}$ and $f_{2}$. Then we obtain with the index transform $l_{1}=r_{1}-k l_{2}$ that

$$
\begin{aligned}
& f_{1}(x)=\sum_{j \geq 0} \sum_{|k| \leq \alpha^{j / 2}} \sum_{l_{2} \in \mathbb{Z}} \sum_{n_{1} \in \mathbb{Z}} \sum_{r_{1} \in I\left(j, n_{1}\right)} c\left(j, k, r_{1}-k l_{2}, l_{2}\right) \alpha^{\frac{3}{4} j} \\
& \times \psi\left(\alpha^{j} x_{1}-\alpha^{j / 2} k x_{2}-r_{1}+k l_{2}, \alpha^{j / 2} x_{2}-l_{2}\right)
\end{aligned}
$$

where $I\left(j, n_{1}\right):=\left\{r \in \mathbb{Z}: \alpha^{j / 2}\left(n_{1}-1\right)<r \leq \alpha^{j / 2} n_{1}\right\}$.

For $j \geq 0$ we set

$$
\begin{aligned}
& a_{j, n_{1}, l_{2}}(x):=\lambda\left(j, n_{1}, l_{2}\right)^{-1} \alpha^{\frac{3+2 K_{1}}{4} j} \sum_{|k| \leq \alpha^{j / 2}} \sum_{r_{1} \in I\left(j, n_{1}\right)} c\left(j, k, r_{1}-k l_{2}, l_{2}\right) \\
& \times \alpha^{-K_{1} j / 2} \psi\left(\alpha^{j} x_{1}-\alpha^{j / 2} k x_{2}-r_{1}+k l_{2}, \alpha^{j / 2} x_{2}-l_{2}\right),
\end{aligned}
$$

if $\lambda\left(j, n_{1}, l_{2}\right) \neq 0$ and $a_{j, n_{1}, l_{2}}(x)=0$ otherwise, where

$$
\lambda\left(j, n_{1}, l_{2}\right):=\alpha^{\frac{3+2 K_{1}}{4} j} \sum_{|k| \leq \alpha^{j / 2}} \sum_{r_{1} \in I\left(j, n_{1}\right)}\left|c\left(j, k, r_{1}-k l_{2}, l_{2}\right)\right| .
$$

By the support assumption on $\psi$, the functions appearing in the definition of $a_{j, n_{1}, m_{2}}$ are only non-zero if the following conditions are satisfied:

$$
-D \leq \alpha^{j / 2} x_{2}-l_{2} \leq D, \quad \alpha^{-j / 2}\left(l_{2}-D\right) \leq x_{2} \leq \alpha^{-j / 2}\left(l_{2}+D\right)
$$

and

$$
\begin{aligned}
-D \leq \alpha^{j} x_{1}-\alpha^{j / 2} k x_{2}-r_{1}+k l_{2} \leq D & \\
\alpha^{-j} r_{1}+\alpha^{-j} k\left(\alpha^{j / 2} x_{2}-l_{2}\right)-\alpha^{-j} D & \leq x_{1} \leq \alpha^{-j} r_{1}+\alpha^{-j} k\left(\alpha^{j / 2} x_{2}-l_{2}\right)+\alpha^{-j} D, \\
\alpha^{-j} r_{1}-\alpha^{-j / 2}(2 D) & \leq x_{1} \leq \alpha^{-j} r_{1}+\alpha^{-j / 2}(2 D), \\
\alpha^{-j / 2} n_{1}-\alpha^{-j / 2}(3 D) & \leq x_{1} \leq \alpha^{-j / 2} n_{1}+\alpha^{-j / 2}(2 D) .
\end{aligned}
$$

Thus, $a_{j, n_{1}, l_{2}}$ is supported in $3 D Q_{j, n_{1}, l_{2}}$, where the cube is considered with respect to $\sqrt{\alpha}$. The appropriate bounds $\left|D^{\gamma} a_{j, n_{1}, l_{2}}\right| \leq \alpha^{\frac{j}{2}}|\gamma|,|\gamma| \leq K_{1}$ can be derived as in the previous proof. Hence the functions $a_{j, n_{1}, l_{2}}$ are $K_{1}$-atoms.

Now we obtain for

$$
f_{1}(x)=\sum_{j \geq 0} \sum_{l_{2} \in \mathbb{Z}} \sum_{n_{1} \in \mathbb{Z}} \lambda\left(j, n_{1}, l_{2}\right) a_{j, n_{1}, l_{2}}(x)
$$


SHEARLET COORBIT SPACES: COMPACTLY SUPPORTED ANALYZING SHEARLETS, TRACES AND EMBEDDING\$ that

$$
\begin{aligned}
\left\|f_{1}\right\|_{B_{p, p}^{\sigma_{1}}}^{p} & \lesssim \sum_{j \in \mathbb{Z}} \alpha^{\frac{j}{2}\left(\sigma_{1}-\frac{2}{p}\right) p} \sum_{l_{2} \in \mathbb{Z}} \sum_{n_{1} \in \mathbb{Z}}\left|\lambda\left(j, n_{1}, l_{2}\right)\right|^{p} \\
& =\sum_{j \in \mathbb{Z}} \alpha^{\frac{j}{2}\left(\sigma_{1}-\frac{2}{p}\right) p} \alpha^{\frac{j}{2}\left(\frac{3+2 K_{1}}{2}\right) p} \sum_{l_{2} \in \mathbb{Z}} \sum_{n_{1} \in \mathbb{Z}}\left|\sum_{|k| \leq \alpha^{j / 2}} \sum_{r_{1} \in I\left(j, n_{1}\right)}\right| c\left(j, k, r_{1}-k l_{2}, l_{2}\right)||^{p} \\
& \leq \sum_{j \in \mathbb{Z}} \alpha^{\frac{j}{2} p\left(\sigma_{1}+\frac{7}{2}+K_{1}-\frac{4}{p}\right)} \sum_{l_{2} \in \mathbb{Z}} \sum_{n_{1} \in \mathbb{Z}} \sum_{|k| \leq \alpha^{j / 2}} \sum_{r_{1} \in I\left(j, n_{1}\right)}\left|c\left(j, k, r_{1}-k l_{2}, l_{2}\right)\right|^{p} \\
& =\sum_{j \in \mathbb{Z}} \alpha^{\frac{j}{2} p\left(\sigma_{1}+\frac{9}{2}+\left\lfloor\sigma_{1}\right\rfloor-\frac{4}{p}\right)} \sum_{|k| \leq \alpha^{j / 2}} \sum_{l_{1} \in \mathbb{Z} l_{2} \in \mathbb{Z}} \sum_{\left|c\left(j, k, l_{1}, l_{2}\right)\right|^{p}} \\
& \lesssim\|f\|_{\mathcal{S C}_{p, r} .}^{p}
\end{aligned}
$$

In the case $j<0$ we obtain with $J\left(j, n_{2}\right):=\left\{r: \alpha^{-j / 2}\left(n_{2}-1\right)<r \leq \alpha^{-j / 2} n_{2}\right\}$ that

$$
\begin{aligned}
f_{2}(x) & =\sum_{j<0} \sum_{l_{1} \in \mathbb{Z}} \sum_{l_{2} \in \mathbb{Z}} c\left(j, 0, l_{1}, l_{2}\right) \alpha^{\frac{3}{4} j} \psi\left(\alpha^{j} x_{1}-l_{1}, \alpha^{j / 2} x_{2}-l_{2}\right) \\
& =\sum_{j<0} \sum_{l_{1} \in \mathbb{Z}} \sum_{n_{2} \in \mathbb{Z}} \sum_{r_{2} \in J\left(j, n_{2}\right)} c\left(j, 0, l_{1}, r_{2}\right) \alpha^{\frac{3}{4} j} \psi\left(\alpha^{j} x_{1}-l_{1}, \alpha^{j / 2} x_{2}-r_{2}\right) \\
& =\sum_{j<0} \sum_{l_{1} \in \mathbb{Z}} \sum_{n_{2} \in \mathbb{Z}} \lambda\left(j, l_{1}, n_{2}\right) a_{j, l_{1}, n_{2}}(x),
\end{aligned}
$$

where

$$
\begin{aligned}
a_{j, l_{1}, n_{2}}(x) & :=\lambda\left(j, l_{1}, n_{2}\right)^{-1} \alpha^{\frac{3-2 K_{2}}{4} j} \sum_{r_{2} \in J\left(j, n_{2}\right)} c\left(j, 0, l_{1}, r_{2}\right) \alpha^{\frac{j K_{2}}{2}} \psi\left(\alpha^{j} x_{1}-l_{1}, \alpha^{j / 2} x_{2}-r_{2}\right), \\
\lambda\left(j, l_{1}, n_{2}\right) & :=\alpha^{\frac{3-2 K_{2}}{4} j} \sum_{r_{2} \in J\left(j, n_{2}\right)}\left|c\left(j, 0, l_{1}, r_{2}\right)\right|
\end{aligned}
$$

and $a_{j, l_{1}, n_{2}}(x):=0$ if $\lambda_{j, l_{1}, n_{2}}=0$. By the support assumption on $\psi$ we get

$$
\begin{aligned}
\alpha^{-j}\left(l_{1}-D\right) & \leq x_{1} \leq \alpha^{-j}\left(l_{1}+D\right), \\
\alpha^{-j / 2}\left(r_{2}-D\right) & \leq x_{2} \leq \alpha^{-j / 2}\left(r_{2}+D\right) \quad \Rightarrow \quad \alpha^{-j}\left(n_{2}-2 D\right) \leq x_{2} \leq \alpha^{-j}\left(n_{2}+D\right) .
\end{aligned}
$$

Consequently, $a_{j, l_{1}, n_{2}}$ is supported in $2 D Q_{j, l_{1}, n_{2}}$. Since $1 \geq \alpha^{j|\gamma| / 2} \geq \alpha^{j|\gamma|} \geq \alpha^{j K_{2}}$ for $0 \leq|\gamma| \leq K_{2}$ and $j<0$ we obtain further that $\left|D^{\gamma} a_{j, n_{1}, l_{2}}\right| \leq \alpha^{j K_{2} / 2} \alpha^{j|\gamma| / 2} \leq \alpha^{j|\gamma|}$ so that $a_{j, l_{1}, n_{2}}$ are $K_{2}$-atoms.. 
Thus,

$$
\begin{aligned}
\left\|f_{2}\right\|_{B_{p, p}^{\sigma_{2}}}^{p} & \lesssim \sum_{j \in \mathbb{Z}} \alpha^{j\left(\sigma_{2}-\frac{2}{p}\right) p} \sum_{l_{1} \in \mathbb{Z}} \sum_{n_{2} \in \mathbb{Z}}\left|\lambda\left(j, l_{1}, n_{2}\right)\right|^{p} \\
& \leq \sum_{j<0} \alpha^{j\left(\sigma_{2}-\frac{2}{p}+\frac{3-2 K_{2}}{4}\right) p} \sum_{l_{1} \in \mathbb{Z}} \sum_{n_{2} \in \mathbb{Z}}\left|\sum_{r_{2} \in J\left(j, n_{2}\right)} c\left(j, 0, l_{1}, r_{2}\right)\right|^{p} \\
& \leq \sum_{j<0} \alpha^{j\left(\sigma_{2}-\frac{3}{2 p}+\frac{1}{4}-\frac{K_{2}}{2}\right) p} \sum_{l \in \mathbb{R}^{2}}|c(j, 0, l)|^{p} \\
& \leq \sum_{j \in \mathbb{Z}} \alpha^{j p r} \sum_{k \in \mathbb{Z}} \sum_{l \in \mathbb{R}^{2}}|c(j, k, l)|^{p} \\
& \lesssim\|f\|_{\mathcal{S C}_{p, r},}^{p}
\end{aligned}
$$

where $r=\sigma_{2}-\frac{3}{2 p}-\frac{1}{4}-\frac{\left\lfloor\sigma_{2}\right\rfloor}{2}$.

Remark 4.8. An alternative way to obtain trace results would be first to apply the Besov embedding and afterwards the classical trace theorem for homogeneous Besov spaces. Let us briefly discuss the relation between these different approaches. For simplicity we restrict ourselves to the positives scales and traces to the $x_{2}$-axis. Usually an application of trace theorems in Besov spaces leads to a loss of smoothness of order $1 / p$, that is $\operatorname{Tr}\left(B_{p p}^{s}\left(\mathbb{R}^{d}\right)\right)=B_{p p}^{s-1 / p}\left(\mathbb{R}^{d-1}\right)$, see [12]. Let the coorbit space smoothness index $r$ be fixed. Depending on the concrete values of $r$ and $p$, the direct and the indirect approach can yield the same result. However, in specific cases it turns out that the direct approach is superior as we gain some smoothness: Let $2 r-\frac{9}{2}+\frac{3}{p}=2 a+\alpha$ with $a \in \mathbb{Z}$ and $\alpha \in[0,2)$. Then we have for $\alpha \in[0,1)$ by Theorem 4.5 that $\sigma_{1}=a+\alpha$. On the other hand, in case $\alpha+\frac{1}{p} \in[1,2)$ an application of Theorem 4.7 yields $\mathcal{S} C C_{p, r} \subset B_{p p}^{\tilde{\sigma}_{1}}$, where $\tilde{\sigma}_{1}=a+1-\varepsilon$ for arbitrary small $\varepsilon>0$. Consequently, applying the trace theorem for Besov spaces yields smoothness $\tilde{\sigma}_{1}-1 / p=a+1-\varepsilon-1 / p<a+\alpha=\sigma_{1}$.

Remark 4.9. Embedding results in Besov spaces have also been shown for the curvelet setting by Borup and Nielsen [4]. However, the technique used by these authors is completely different. In contrast to our approach they work in the frequency domain. We prefer to consider the time domain with flexible atomic decompositions for the following reasons. As already outlined above time domain techniques provide a very natural way to derive trace theorems which might be very difficult or even impossible in the Fourier domain. Moreover, since we are working with compactly supported atoms the treatment of shearlet coorbit spaces on bounded domains, including again embedding and trace theorems, seems to be manageable. We also think that our approach provides some advantages for higher dimensions. One natural conjecture would be that the traces of shearlet coorbit spaces on $\mathbb{R}^{3}$ with respect to two-dimensional hyperplanes are again shearlet coorbit spaces. To prove this conjecture, again flexible atomic decomposition techniques for shearlet coorbit spaces would be needed. By following the lines corresponding to the Besov space setting there is some hope that such flexible decompositions can be derived. These questions will be discussed in forthcoming papers. 


\section{Acknowledgement}

The authors warmly thank Karlheinz Gröchenig and Dorothee Haroske for private lessions in coorbit and function space theory, respectively. For pointing our attention to the topic of remark 4.8 we thank P. Grohs. The authors further acknowledge partial support by the Deutsche Forschungsgemeinschaft Grants DA 360/12-1,13-1, STE 571/9-1, TE 354/5-1 and TE 354/8-1.

\section{REFERENCES}

[1] R.A. Adams, Sobolev Spaces, Academic Press, Now York, 1975.

[2] E.J. Candes and D.L. Donoho, Ridgelets: a key to higher-dimensional intermittency?, Phil. Trans. R. Soc. Lond. 357, 2495 - 2509 (1999).

[3] E.J. Candes and D.L. Donoho, Curvelets - A surprisingly effective nonadaptive representation for objects with edges, in : Curves and Surfaces, L.L. Schumaker et al., eds, Vanderbild University Press, Nashvill, TN (1999).

[4] L. Borup and M. Nielsen, Frame decomposition of decomposition spaces, J. Fourier Anal. Appl. 13/1, 39 - 70 (2007).

[5] S. Dahlke, G. Kutyniok, P. Maass, C. Sagiv, H.-G. Stark, and G. Teschke, The uncertainty principle associated with the continuous shearlet transform, Int. J. Wavelets Multiresolut. Inf. Process., 6, 157 - 181 (2008).

[6] S. Dahlke, G. Kutyniok, G. Steidl, and G. Teschke, Shearlet coorbit spaces and associated Banach frames, Appl. Comput. Harmon. Anal. 27/2, 195 - 214 (2009).

[7] S. Dahlke, G. Steidl, and G. Teschke, The continuous shearlet transform in arbitrary space dimensions, J. Fourier Anal. App. 16, 340 - 354 (2010).

[8] H. G. Feichtinger and K. Gröchenig, A unified approach to atomic decompositions via integrable group representations, Proc. Conf. "Function Spaces and Applications", Lund 1986, Lecture Notes in Math. 1302 (1988), 52 $-73$.

[9] H. G. Feichtinger and K. Gröchenig, Banach spaces related to integrable group representations and their atomic decomposition I, J. Funct. Anal. 86, 307 - 340 (1989).

[10] H. G. Feichtinger and K. Gröchenig, Banach spaces related to integrable group representations and their atomic decomposition II, Monatsh. Math. 108, 129 - 148 (1989).

[11] K. Gröchenig, Foundations of Time-Frequency Analysis, Birkhäuser, Boston, Basel, Berlin, 2001.

[12] M. Frazier and B. Jawerth, Decomposition of Besov sapces, Indiana University Mathematics Journal 34/4, 777 - 799 (1985).

[13] K. Gröchenig, Describing functions: Atomic decompositions versus frames, Monatsh. Math. 112, 1 - 42 (1991).

[14] K. Guo, G. Kutyniok, and D. Labate, Sparse multidimensional representation using anisotropic dilation and shear operators, in: Wavelets and Splines (Athens, GA, 2005), G. Chen and M.J. Lai, eds., Nashboro Press, Nashville, TN (2006), 189-201.

[15] L.I. Hedberg and Y. Netrusov, An axiomatic approach to function spaces, spectral synthesis, and Luzin approximation, Memoirs of the American Math. Soc. 188, 1- 97 (2007).

[16] P. Kittipoom, G. Kutyniok, and W.-Q Lim, Construction of compactly supported shearlet frames, Preprint, 2009.

[17] G. Kutyniok and D. Labate, Resolution of the wavefront set using continuous shearlets, Preprint, 2006.

[18] G. Kutyniok, J. Lemvig, and W.-Q. Lim, Compactly supported shearlets, Preprint, 2010.

[19] C. Schneider, Besov spaces of positive smoothness, PhD thesis, University of Leipzig, 2009.

[20] H. Triebel, Function Spaces I, Birkhäuser, Basel - Boston - Berlin, 2006

Philipps-Universität Marburg, FB12 Mathematik und Informatik, Hans-Meerwein Strasse, LahnBerge, 35032 Marburg, Germany

E-mail address: dahlke@mathematik.uni-marburg.de

Universität Mannheim, FAKultät FÜr Mathematik Und Informatik, Institut für Mathematik, 68131 Mannheim, Germany

E-mail address: steidl@math.uni-mannheim.de

Hochschule Neubrandenburg - University of Applied Sciences, Institute for Computational Mathematics in Science and Technology, Brodaer Str. 2, 17033 Neubrandenburg, Germany

E-mail address: teschke@hs-nb.de 